African Research Review

An International Multidisciplinary Journal, Ethiopia

Vol. 10(1), Serial No.40, January, 2016: 243-256

ISSN 1994-9057 (Print)

ISSN 2070--0083 (Online)

Doi: http://dx.doi.org/10.4314/afrrev.v10i1.19

\title{
Les Problèmes Linguistiques, Culturels et Sociolinguistiques De La Traduction Du Film "Winning Your Love" Par Ossy \\ Affason
}

\author{
Asadu, Oluchukwu Felicia \\ Department of Modern European Languages \\ Nnamdi Azikiwe University, Awka \\ Anambra State, Nigeria \\ E-mail: asaduoluchukwu@yahoo.com \\ Phone: +2348038740016
}

\section{Résumé}

Le film dont on parle est un film à long métrage. Ce n'était pas facile de trouver le manuscrit du film, mais là, où on l'a trouvé, le contenu était différent de ce que disaient les acteurs. Nous avons écouté la sonorité du film qui exige la spontanéité afin de le mettre sur papier et puis le traduire. Nous avons rencontré beaucoup de problèmes à repérer les termes difficiles ou les tournures particulières. Au cours de la traduction de ce film nous avons identifié des problèmes linguistiques, culturels, et sociolinguistiques. Le film "Winning your love" est un film nigérian et comme beaucoup de films montés au Nigéria, le problème linguistique est majeur. Le Nigéria, un pays anglophone est tout d'abord un pays de beaucoup d'ethnies (on dit environ 300) dont les langues sont les Haoussa, les Yoruba, et les Igbo. Beaucoup de Nigerians ne parlent l'anglais qu'avec beaucoup d'interférence de leurs langues locales. Pour 
discuter les problèmes linguistiques, nous allons voir les problèmes lexicographiques, sémantiques, sociolinguistiques, métalinguistiques, morphologiques et syntaxiques.

\section{Introduction}

\section{Le film comme une création littéraire}

Les mouvements de la caméra, les effets spéciaux, les trucages cinématographiques, et la rigueur du montage font du film un art qui maitrise parfaitement le temps narratif. Un film cherche à exprimer des émotions profondes, une représentation nouvelle de la réalité et un découpage du temps réel. Le film fait partie de la traduction littéraire. La traduction littéraire consiste en trois genres majeurs de la littérature. Le film est tout simplement la présentation des images désignant les actions des personnages. Dans le théâtre, il y a des personnages qui jouent sur scène en imitant la vie réelle.

Dans le film et aussi au théâtre, on a toujours des acteurs qui jouent des rôles des personnages. Opposé au théâtre, le film est enregistré sur bande magnétique et joué à l'écran. On peut avoir un film en long métrage ou en court métrage. La seule différence entre le film et le théâtre c'est que le film consiste en photos des hommes sur l'écran tandis qu'au théâtre le spectacle consiste des hommes sur scène. Le schéma c'est ainsi :

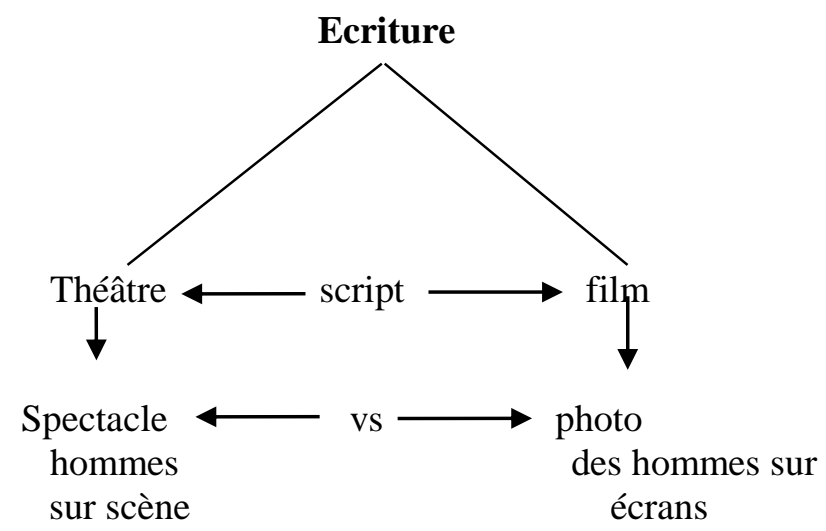

Il y a différents types de traduction orale comme la narration, les anecdotes, les dialogues, les poésies orales et les représentations dramatisées. Un film peut avoir toutes ces formes mentionnées. Un film est une chose brève. Parfois on entend des expressions verbales accompagnées par des gestes, des mimiques, des chansons ou les inexprimés dans la traduction. Ces inexprimés aident les sens que donnent les expressions verbales.

C'est pourquoi Ezeafulukwe (2005:51) dit ainsi: 
Le traducteur de film se voit donc face aux problèmes de l'incompréhension des gestes faits par les acteurs qu'il doit faire comprendre à l'audience. Il se garde contre la surcharge d'informations tout en respectant l'esprit de la spontanéité qui est caractéristique du genre oral.

\section{Problèmes Linguistiques}

Le film "Winning your love" est un film nigérian et comme beaucoup de films montés au Nigéria, le problème linguistique est majeur. Le Nigéria, un pays anglophone est tout d'abord un pays de beaucoup d'ethnies (on dit environ 300) dont les langues sont les Haoussa, les Yoruba, et les Igbo. Beaucoup de Nigerians ne parlent l'anglais qu'avec beaucoup d'interférence de leurs langues locales. Pour discuter les problèmes linguistiques, nous allons voir les problèmes lexicographiques, sémantiques, sociolinguistiques, métalinguistiques, morphologiques et syntaxiques.

\section{Problèmes Lexicographiques}

Ce sont des problèmes que le traducteur peut avoir avec l'usage des mots. Parfois quelques expressions sont mal utilisées.

Par exemples

i. TLD 90: ...I learnt she is in the campus as well.

TLA 90: ...j'ai appris qu'elle est aussi à l'université.

Cette phrase manque la préposition adéquate et aussi n'a pas besoin d'article.La phrase correcte serait $I$ learnt she is an undergraduate.

ii TLD $531: \ldots$ you think I'm up before.

TLA 531 ...tu crois que je ne sais pas ce que je fais.

Le mot « up » dans la version anglaise est implicite est aussi mal placé mais la version française est plus explicite « up » vaut dire « haut » mais ici cela vaut dire « $\mathbf{u n}$ fou » à travers le dialogue.

iii TLD 464 :...I am doing my best.

TLA 464: ....Je fais de mon mieux pour la réussite.

On a une explicitation du mot « best « en anglais. Le mot « best » serait être traduit comme « la meilleure » mais, pour que le sens soit clair à l'audience, on a fait l'explicitation parce que, selon le contexte, l'auteur fait référence à la réussite à l'éxamen. 


\section{Problèmes Sémantiques}

On constate parfois des expressions qui ne peuvent pas être comprises dans d'autres milieux anglophones mais qui sont comprises dans un milieu anglophone nigérian.

Par exemples:

i TLD $266: . .$. what authentic bullshit?

TLA 266: ...quel merde!

Le mot authentic n'a rien en commun avec bullshit. Donc on a traduit le sens.

ii TLD 26:... if the job is ready for me, then I will jump to it.

TLA 26: ...si le travail est là pour moi, je dois commencer tout de suite.

« Je saute sur le travail » est une figure de style non soutenue.

iii TLD $12: \ldots$ Eh brother Douglas.

TLA 12: ...Oh! Bienvenu grand frère Douglas.

En français il faut un adjectif qualificatif pour déterminer frère, pour le distinguer d'un frère religieux

\section{Problèmes Syntaxiques}

Quelques phrases utilisées dans le texte sont soit agrammaticales soit dépourvues de sens. Des phrases comme les suivantes sont difficiles à comprendre à cause de ces fautes.

i TLD $376:$ :..l'll not stop on the other part that beat your love.

TLA 376: ....je ne supporte pas l'adoration de l'argent.

Cette phrase est bien comprise parmi le groupe en question.

ii TLD 155: ....real love ushers durability.

TLA 155: ...c'est seulement le vrai amour qui dure.

Le verbe « usher » est suivi toujours par une préposition «in » en anglais et il prend un objet aussi.

iii TLD 205: ... you continually collaborated my impression of you.

TLA 205: ...tu continues à confirmer mes impressions sur toi.

Le verbe « collaborate » va avec « with » en anglais et pas avec un pronom.

iv TLD 30: ....aren't you making things so easy for me ? 
TLA 30: ...tu me gâtes...

Un adjectif en anglais traduit comme un verbe en langue française. Le mot easy est un adjectif traduit comme un verbe à l'indicatif présent " gâtes ».Une phrase interrogative en anglais traduite comme une phrase declarative en français.

v TLD 296: You should have seen...

TLA 296: Tu aurais dû voir...

Le participe passé seen se voit traduire avec un verbe infinitif en français voir. Pour maintenir la structure française.

\section{Problèmes Sociolinguistiques}

Ce sont les problèmes qui se présentent au milieu social d'usage linguistique. Naturellement un individu s'exprime différemment dans une langue selon des contextes sociaux. D'ailleurs, sa façon de s'exprimer montre son origine sociale ou nationale. Parmi ces problèmes nous avons relevé les suivants:

i TLD 51: Give him water to drink.

TLA 51: Donne-lui à boire.

Dans ce context le Français n'a pas besoin de mentionner l'objet parce qu'il a déjà commencé à manger.

ii TLD 138: ...baby.

TLA 138: ...chérie.

Naturellement le mot «baby » veut dire « bébé » en français mais, en tenant compte du contexte social du mot, nous l'avons traduit comme « chérie ».

iii TLD 616: Honey...

TLA 616: mon chéri...

Selon notre explicitation ci-dessus « honey » qui vaut dire « miel » ici vaut dire « mon chéri » en nous respectant le contexte sociolinguistique où il se trouve.

iv TLD 108: Alright, fine, you stay cool.

TLA 108: Au revoir.

En Français on utilisé seulement un mot pour remplacer toutes les formules dé séparation; c'est-à-dire les formules pour prendre congé de quelqu'un.

v TLD 42: Man...

TLA 42: mon vieux... 
Le mot est traduit par une expression équivalente. « Man » est un mot familier utilisé entre égaux intimes. Son équivalent en français est « mon vieux », utilisé dans la même situation d'égalité et d'amitié.

vi TLA 48: You mean I go wait.

TLA 48: C'est-à-dire je vais attendre.

Cette version anglaise est argotique et acceptable au Nigéria. Nous l'avons traduit en français standard car c'est un exercice académique.

\section{Problèmes Morphologiques} Exemples:

Ce sont des problèmes qui se posent par l'unité minimale de signification.

I TLD 180: ...i'm running late.

TLA 180: ...je suis pressée.

Le mot late en anglais est un nom traduit comme un verbe être pressé. Ici on voit que la version anglaise n'a pas besoin d'accord mais en français c'est obligatoire pour faire l'accord là ou il est nécessaire, c'est pourquoi on double le e du mot pressé pour montrer le genre et le nombre de personne qui parle. Ce phénomène n'existe pas en anglais. Voilà un des problèmes auxquels le traducteur fait face.

ii

TLD 40: You...

TLA 40: Tu ...

En anglais on a une seul forme de « you » qui est un pronom personnel de deuxième personne singulier ou pluriel tandis qu'en français le pronom « you « a deux formes qui sont «tu » ou « vous » selon la personne qui parle.

« $\mathbf{T u} »$ s'utilise entre amis et en famille tandis que « vous » est une forme de politesse ainsi que le pluriel.

iii TLD 43: The meals...

TLA 43: Les recettes...

En anglais, on a seul article défini " the » mais en français le nom s'accorde avec le nombre. C'est pourqoui nous avons « les».

\section{Problèmes Métalinguistiques}

Ce sont des problèmes qui se trouvent dans la traduction des mots anglais en langue française ou vice versa. Le traducteur parfois rencontre ces mots dont il ne connait pas la racine. Par exemple: 


\section{TLD 112: ...business.}

\section{TLA 112: ...mon business?}

«Business » est un emprunt de l'anglais au français et ce mot est utilisé en français pour parler d'affaires mais avec des sens différents.

ii TLD 71: Mum.

\section{TLA 71: Maman.}

En anglais on dit « mum »qui est un mot abrégé pour dire mummy mais en français cela n'existe pas, on dit maman chaque fois on fait référence à une mère.

iii TLD 108: ... since it is weekend.

TLA 108: ...puisque c'est le weekend.

Le mot «weekend » est un mot anglais emprunté à la langue française tel qu'il est pour parler de la fin de semaine.

iv TLD 460: ... its bizarre man.

TLA 460: ... c'est bizarre!

Le mot «bizarre » est un mot français emprunté à l'anglais pour décrire quelque chose d'insolite.

\section{Problèmes Culturels.}

La langue Igbo est une langue bourrée des proverbes. On dit en Igbo qu'un enfant auquel on adresse un proverbe et l'explique aussi, la dot de sa mère est comme une perte d'argent.

« Nwata a tụọọ ilu kọwara ya ego e jiri lụa nne ya lara n'iyi. »

Mais les proverbes sont utilisés pour des raisons différentes et font appel à plusieurs associations pour être bien interprétées. Elles se prônent alors à plusieurs interprétations parfois susceptibles aux interprétations erronées.

Le traducteur étant un être imparfait peut tomber dans le piège d'une mauvaise interprétation du proverbe du texte, faute de ne pas bien faire des associations nécessaires.

Par exemple:

i TLD 365: ... he who wants vegetable must first pray for a good rain.

TLA 365: ...comme on fait son lit, on se couche. 
TLD 314: ... a man that is a few inches away from his grave.

TLA 314: ... un vieux qui est déjà près de son tombeau.

La langue véhicule toute une culture. Donc la traduction d'une langue à l'autre ne peut qu'être approximative car chaque pensée est une abstraction et la langue ne fait qu'expulser des pensées. Ainsi les pensées sont conçues et modelées selon des expériences vécues et entendues. L'émetteur émet alors toute une culture qui lui est propre. Le traducteur ne fait que saisir ce qu'il peut et ne traduit que ce qu'il a pu saisir.Saisir un proverbe autochtone mais rendre le message saisi dans une autre langue (une autre culture) n'est pas une tâche facile. Le traducteur étant anglophone a tenté cette traduction en tant que traducteur dans la troisième langue. Le cas de double traduction dans la traduction des œuvres africaines est une chose particulière. Les pays africains sont d'abord multilingues. Ils ont des langues européennes comme langues officielles. Dans les œuvres produites par les Africains comme le dirait Senghor. "Nous nous sentons en nègres mais nous exprimons en français ». Les Africains conçoivent leurs œuvres en langues africaines et les traduisent en langues européennes et le traducteur les traduit encore en une troisième langue. Beaucoup de gains et de pertes d'informations sont effectués au cours de ce processus. Parfois ni artiste ni le traducteur ne s'en rende compte. Exemples:

iii TLD 289: ... and pushed me down on the floor in my own husband's house.

TLA 289: ....m'a fait tomber par terre dans la maison de mon mari.

La connotation de cette traduction entraine des croyances sociales et culturelles où il est estimé déjà coupable celui qui vient bagarrer dans la maison de quelqu' un d'autre. La culture igbo donne un droit inexprimé au propriétaire de la maison de se défendre de toute manière possible une fois un ennemi le pousse jusqu' à chez lui parce que le droit de propriétaire sera en sa faveur pour gagner l'ennemi, même sans tenir compte de la raison du bagarre.

iv TLD 357: Madame Tension ọkụ.

TLA 357: Madame Haute Tension.

C'est un fait culturel que des gens portent des noms qui s'alignent à leurs comportements. Nous constatons Mme Tension qui porte ce nom à cause de son comportement qui a le même effet avec un effet paralysant, choquant et immédiate de la Haute Tension électrique. Mme. Tension se vante avec ces attributs auxquels elle met d'emphase à 1 aide de son équivalent Igbo. Le traducteur, à son tour trouve la nécessité de reprendre cette emphase mais se sert maintenant de son équivalent anglais pour son emphase au lieu de l'igbo du texte.

v TLD 238: ...no sacrament that binds us. 
TLA 238: ... entre le vautour et la coiffeuse, rien à cirer.

L'idiome du départ reçoit ici une traduction qui se distingue en forme linguistique mais qui maintient une congruité sémantique avec le vouloir-dire original. Des idiomes ne prennent de sens qu'en function du contexte. Un même idiome peut donc s'adapter à plusieurs situations en exprimant des visés différents. Voilà la rasion pour laquelle la traduction de cet idiome peut passer par d'autres formes linguistiques sans se cogner au sens.

Nous voyons encore Madame Tension qui ponctuait toujours ses paroles avec la langue indigène Igbo. Nous entendons les mots comme, $O$ wụ ashị, I gbulifụ m etc. Bienque le Nigeria soit un pays anglophone, les langues maternelles dominent toujours dans des milieux non-officiels. Ces emprunts ajoutaient au style des personnages et du producteur. Mais ce style est mieux apprécié dans un milieu nigérian où les deux langues: anglaise et Igbo sont comprises, par les cinéphiles mais la traduction de ce film en français vise à un autre groupe de cinéphiles. Des cinéphiles non nigérians qui ne comprendraient rien de ces mots vernaculaires risquent de perdre un peu de sens si ces emprunts restent. Il a fallu alors que nous traduisions quelques uns de ces emprunts qui normalement devraient retenir leurs formes originales. Exemples: Oggo bụ chi onye, alu emee $\mathbf{m}, \mathbf{0}$ ka $\mathbf{m}$ si jee pour la simple raison de rester fidèle au sens premier.

TLD 765: ...ọgọ bụ chi onye

TLA 765: ....mon petit fils aimé (mon petit dieu)

Au milieu Igbo, cette expression est bien comprise et est beaucoup utilisée car une croyance existe qui soutient qu'un gendre de soi est pour lui un petit dieu puisqu' il peut faire ou défaire la vie de la famille de sa femme. Un gendre riche apporte la richesse tandis qu'un gendre pauvre apporte aussi la pauvreté. Cette croyance souligne pourquoi on doit se méfier dans les choix des gendres et pourquoi cette relation bien précaire doit être considéré avant d'accepter le prétendant. Une situation pareille n'existe pas en français, ce qui a informé l'explicitation "mon petit dieu", ajouté après l'expression empruntée pour mieux éclairer le contexte social et la connotation culturelle de cette expression calquée qui peut ne rien dire aux non -igbophones.

vii TLD 601: ....alụ emee m !

TLA 601: ...je suis fichu.

C'est toute une culture igbo qui est véhiculée à travers l'expression de départ. «Alu emee m » veut dire. "Une chose abominable" n'est annulée. Cette expression est utilisée quand les choses ne marchent pas comme prévus. Une chose abominable est pourtant conçue de manières différentes par des gens différents. Le traducteur laisse le locateur déduire cette chose abominable à sa manière./

viii TLD 603: Ọ ka m si jee. 
TLA 603: Voilà ce qui m'arrive.

La même chose s'applique ici. C'est toujours une expression figée qui connote le désespoir total est traduit avec un simple. "Voilà ce qui m'arrive".Les deux expressions se vêtissent du sens une fois le contexte établi. La version originale semble plus lourde tandis que la version française est plus légère.

ix TLD 96: Tụfiakwa!

TLA 96: Jamais de la vie!

Une exclamation Igbo qui exprime le rejet et le dégout est traduite en français comme Jamais de la vie! Qui exprime aussi un rejet et un dégout pour que les nouveaux cinéphiles le comprennent. La musique de fond est en Igbo. Elle est jouée pour animer les scènes. Elle aide alors à comprendre et à prévoir les scènes à venir. Avec le sous- titrage, ces chansons restent en Igbo et parce que ce n'était pas possible de les traduire.

\section{Problèmes Extralinguistiques}

Nous avons constaté le problème de synchroniser les mots avec les actions et l'image. C'était très difficile de traduire les inexprimés comme des gestes, des mimiques, l'intonation, les signes et quelques expressions figées. Ce que nous avons fait c'est de fuser les exprimés et les inexprimés véhiculés par la forme pour réaliser un film plus proche de l'orignal.Aussi pour réaliser la synchronisation, il nous faut un spécialiste.

\section{Les Problèmes des Non-dits}

Les gestes et les mimiques dans le texte sont utilisés pour exprimer beaucoup de choses. Nous voyons par exemple la rencontre de Mme Tension avec Douglas. Douglas qui s'est présenté comme secrétaire d'une compagnie n'arrive pas à gagner le respect de Madame Tension qui conçoit le travail du secrétaire comme celui d'un simple agent de courrier qui ne fait que classer les dossiers. Elle décrit un secrétaire avec dédain en ces mots. File arrangement clerk? (353)/...qui classe des documents! (353). Sa conception du secrétaire l'importune et lui ferme l'esprit contre Douglas.

Même au moment où sa fille présentait Douglas, elle montrait déjà par les mouvements de ses yeux combien il était ignoble avant même de commencer à s'exprimer. Elle avait tout dit par ses gestes et ses mimiques. Néanmoins, la technique soutitrage ne resoud pas ces problèmes.

Problèmes Sociologiques: ce sont des problèmes le traducteur fait face parce que les habitudes des gens, des sociétés se manifestent dans leurs expressions. Alors, le traducteur doit s'habituer aux cotextes sociologiques pour surmonter ces problèmes. 


\section{TLA 292: À Dieu ne plaise}

Nous constatons ici, dans la traduction, une expression figée qui rend exactement la situation dans la langue source. Il y a donc une équivalence de situation mais exprime à travers des formes linguistiques entièrement différentes.

ii TLD 296: ... she really pissed me off

TLA 296: ... elle m'a vraiment énervée

La traduction est telle que ce n'est que le sens saisi par le traducteur qu'il traduit. L'expression de la langue de départ est une phrase familière, utilisée parmi les jeunes pour exprimer l'irritation. La traduction présente l'effet de la phrase au lieu de sa cause. iii TLD 283: I'm embrassing you.

TLA 283: Tu oses dire que je t'embarrasse.

Notons ici une traduction où le sentiment qui pousse le locuteur est mis en jeu pour traduire sa parole. Les mots du texte et les mots de la traduction ne sont_donc pas pareils.

iv TLD 601: Heee!

TLA 601: Oh la la! (601).

Ici nous avons à faire avec une exclamation de malaise exprimée en deux formes différentes. La traduction des exclamations sont sociologiques. Une traduction linguistique heurtera au génie de la langue.

v TLD 541: ... get the key for casting.

TLA 541: ... à fin qu'on puisse achever notre but.

Il a fallu une explicitation de l'expression originale pour passer le message au lecteur. Les deux phrases de travail ne partagent ni la similarité lexicale ou sémantique mais sociologique.

vi TLD 126: ... I will slap the hell out of your eyes.

TLA 126: ...je vais bien te gifler.

La version anglaise est idiomatique mais la version française a perdu cette qualité. Elle est traduite simplement sans le poids nécessaire. Il a donc fallu des actions des films pour remettre encore ce poids pour avoir l'effet que cherche le cinéaste. 
Nous voyons aussi combien d'argent le Chef Benson gaspillait sur Madame Tension. Il ne pensait que l'argent lui gagnerait l'amour qu'il cherchait pour épouser Obumneme; une pensée qui s'est prouvée erronée vers la fin du film.

\section{Solutions aux Problèmes}

Les problèmes mentionnés en haut se trouvent dans les deux techniques de traduire un film à savoir: le doublage et le sous-titrage. Pour éviter ces problèmes, le traducteur est obligé de s'internaliser et de s'intégrer d'avantage dans la culture et les pratiques quotidiennes du film en question. C'est pourquoi Asadu (2012: 155) a dit:

Il faut aussi que le traducteur d'un film sache qu'un film est fait non seulement de mots mais aussi de la culture. En d'autres termes il faut que le traducteur regarde le film plusieurs fois et comprenne le sens caché d'un énoncé et l'entourage des expressions avant de traduire. Il ne $\mathrm{s}$ agit pas de traduire directement mot à mot mais de saisir l'idée avant de ré exprimer; parce qu'un film mal traduit aboutira au non sens et pourrait normalement conduire le spectateur à rejeter le film.

Pour dégager le sens d'un film, nous avons utilisé la théorie interprétative préconisée par Danica Seleskovitch et Lédérer mais pour l'analyse d'un film nous avons utilisé les procédés variées.Le traducteur d'un film doit savoir qu' il ne s'agit pas simplement de trouver une bonne traduction qui exprime en tout le sens de la réplique naturelle mais il faut aussi que cette traduction soit synchronique avec le mouvement des lèvres des acteurs à l'écran tout en respectant les appuis de ces acteurs. Pour supporter ceci Asadu (2009:363) a dit $:<$ Le traducteur doit être fidèle à l'œuvre, au niveau de langue, subtilités de l'intrigue tout en étant cohérent des tutoiements et vouvoiements entre les personnages pour donner qu'un exemple.>

\section{Conclusion}

La traduction d'un film reste un sine qua non à la communication efficace car le film se compose de l'orale et de l'écrit aussi. Beaucoup de nos acteurs et de nos actrices seront connus ailleurs si nous traduisons nos films nigérians tandis que les producteurs vont bénéficier des devises étrangères.

Wole Soyinka, Chinua Achebe et leurs contemporains sont renommés, grâce à la traduction de leurs œuvres dans la langue étrangère, ce qui manque chez les acteurs nigérians. Prenant l'Inde comme un exemple; beaucoup de ses films ne sont pas traduits en d'autres langues pour être apprécié par la majorité du peuple du monde. Selon Okhakhu (1991:67):

India produces an average of over nine hundred (900) feature films, one thousand three hundred $(1,300)$ short documentary films, one hundred (100) series and six thousand $(6,000)$ advertisement films 
every year. There are nearly twelve thousand and five hundred $(12,500)$ cinema theaters in India with a total seating capacity of 13 million, Indian films are exported to over one hundred (100) countries in the world, and in 1985 alone, India exported film worth 6 millionUS dollars (67).

L'Inde réalise une moyenne de plus de neuf cent (900) films à long métrage, mille trois cent $(1,300)$ films documentaires cent $(100)$ séries et six mille films publicitaires chaque année. Nous avons presque douze mille cinq-cents $(2,500)$ amphithéâtres en Inde avec un plan de table de treize million au total.... Les films indiens sont exportés à plus de cent(100) pays dans le monde, et en mille neuf cent quatre vingt cinq(1985) par exemple, L'Inde a exporté des films qui valent six million de dollars américains. (Notre traduction).

\section{Références}

Asadu, F.O. <Les problèmes a la traduction du film in MUKABALA.Vol.2 No.2 pp3563712009.

--- <Une traduction commentée (anglais-français) du film Winning Your Love par Ossy Affason.Memoire pour l'obtention du doctorat.Diss Abia State University, UturuOkigwe, 2012.

Chuquet, Helène, et Paillard Michel, Approche linguistique des problèmes de traduction.Paris: Ophrys, 1987.

Delisle, J. L'Analyse du discours comme méthode de traduction 2nd. Edn. Ottawa: Presses de l'Universite d'Ottawa, 1982.

Ezeafulukwe, O.U. "Vers une traduction du film «Amina » Mémoire pour l'obtention de la Maitrise és lettres.Diss: University of Nigeria, Nsukka, 2005.

Gregory, M. and S Carrol Language and Situation: Language Varieties and Their Social Contexts, London: Routledge and Kegan Paul, 1978

Okhakhu, Michael "Film making is capital intensive" in The Role of the Film Industry Jos: NUTAF 1991.

Vinay, J. P. \& Darbelnet, J. Stylistique comparée du français et de l'anglais. Paris: Didier, 1977.

Dictionnaire encyclopédique Larousse. Paris: Larousse, 1990.

Dictionary of Translation Studies. Manchester: St Jerome, 1997.

Dictionnaire de linguistique. Paris: Larousse1991. 
AFRREV, 10 (1), S/NO 40, JANUARY, 2016

Ezeafulukwe, O.U. "Vers une traduction du film « Amina » Mémoire pour l'obtention de la Maitrise és lettres.Diss: University of Nigeria, Nsukka, 2005.

Delisle, J. L'Analyse du discours comme méthode de traduction 2nd. Edn. Ottawa: Presses de l'Universite d'Ottawa, 1982. 